\title{
Some classes of statistical distributions. Properties and Applications
}

\author{
Irina Băncescu
}

\begin{abstract}
We propose a new method of constructing statistical models which can be interpreted as the lifetime distributions of series-parallel/parallelseries systems used in characterizing coherent systems. An open problem regarding coherent systems is comparing the expected system lifetimes. Using these models, we discuss and establish conditions for ordering of expected system lifetimes of complex series-parallel/parallel-series systems. Also, we consider parameter estimation and the analysis of two real data sets. We give formulae for the reliability, hazard rate and mean hazard rate functions.
\end{abstract}

\section{Introduction}

Recently, several methods for deriving new parametric families of probability distributions attracted a special interest, due to their use for the development of wider statistical applications. Transmutation maps [18] represent techniques for introducing skewness or kurtosis into a symmetric or other distribution. They are based on the functional composition of the cumulative distribution function of one distribution with the inverse cumulative distribution function of another. This method can be used in Monte Carlo simulations and

Key Words: expected system lifetimes, multiple transmuted distributions, goodness-offit, stochastic ordering, complex serie-parallel/parallel-series systems.

2010 Mathematics Subject Classification: Primary 62E10; Secondary 62N05, 62E15, $62 \mathrm{P} 30$.

Received: 31.03 .2016

Revised: 23.06.2017

Accepted: 29.06.2017 
copula applications. Parametric distributions which can be obtained by transmutation include the skew-uniform [15], skew-exponential, skew-normal and skew-kurtosis-normal [18]. Many statisticians have used transmutation maps, but especially the quadratic rank transmutation map, applied to generalize well-known distributions. We can mention here the transmuted exponentiated exponential distribution introduced in 2013 by Merovci [11], the transmuted Pareto distribution, proposed in 2014 by Merovci and Puka [12] and many others. The rank transmutation map is used as a tool for generating new families of non-Gaussian distributions, by modulating a given base distribution with the aim of modifying its moments, in particular skewness and kurtosis.

Coherent systems have been so far characterized using signatures [14]. In this paper, by using the quadratic rank transmutation map, we construct a new class of skewed distributions, obtained by multiple application of the transmutation method. These new statistical models represent the lifetime distributions of complex series-parallel/parallel-series systems. An example of a seriesparallel system is the E-17-AH bridge from Colorado, USA [16, 9]. Multi-girder bridges are characterized by these types of systems $[16,2,9]$. Other distributions that represent the lifetime distributions of series-parallel/parallel-series systems can be found in [13] and [7]. These few statistical models represent only a particular and small class of series-parallel/parallel-series systems. Using the method presented in this paper, we can represent a larger class of these type of systems. We cannot represent all the series-parallel/parallelseries systems using the method introduced in this paper, only a particular class of systems. These particular series-parallel/parallel-series systems have independent and identically distributed components.

Choosing the optimal structure design system can be difficult. One tool that helps us in this matter is the expected system lifetime, the simpler and most commonly used metric for system performance [14]. The problem of comparing system performance using the expected system lifetime is still an open problem. Boland and Samaniego (2004) [8] discussed this problem providing conditions for ordering the expected system lifetimes for a particular group of small systems. "Eventhough this comparison ignores the variability in system lifetime it is possible that a system whose expected lifetime exceeds that of a second system will be less reliable than the second system at the systems planned mission time" [14]. This paper discusses the stochastic ordering, namely the likelihood ratio ordering, of some particular series-parallel/parallelseries systems and provides an example.

The paper is organized as follows. In Section 2, the multiple transmuted models of order $n$ are introduced. Section 3 is dedicated to characteristics of these new models. We give formulae for the reliability, hazard rate and mean residual life functions. We discuss the order statistics and their asymptotic 
behaviour. Stochastic ordering is discussed in Section 4, while in Section 5 we discuss the maximum likelihood estimators of the parameters of the new classes of statistical models introduced. We perform data analysis using two real data sets in Section 6 .

\section{Method of construction}

Using the transmutation map developed by Shaw and Buckley (2007) [18], we define the class of transmutated distributions of order $n$. The rank transmutation map for a baseline continuous distribution function $G$ is defined as

$$
F_{T}(x)=(1+\lambda) G(x)-\lambda G(x)^{2}
$$

where $|\lambda| \leq 1$ is the transmutation parameter.

The corresponding density function of $F_{T}$ is

$$
f_{T}(x)=g(x)[1+\lambda-2 \lambda G(x)]
$$

where $g$ is the density function corresponding to $G$.

The class of transmutated distributions of order $n$, denoted by $T_{n}$, is defined as follows. Let $F$ be an arbitrary continuous cumulative distribution function and

$$
\begin{aligned}
& T_{1}=T_{1}\left(F, \lambda_{0}\right): \quad F_{1}(x)=\left(1+\lambda_{0}\right) F(x)-\lambda_{0} F(x)^{2} \\
& T_{2}=T_{2}\left(F, \lambda_{0}, \lambda_{1}\right): \quad F_{2}(x)=\left(1+\lambda_{1}\right) F_{1}(x)-\lambda_{1} F_{1}(x)^{2} \\
& T_{3}=T_{3}\left(F, \lambda_{0}, \lambda_{1}, \lambda_{2}\right): \quad F_{3}(x)=\left(1+\lambda_{2}\right) F_{2}(x)-\lambda_{2} F_{2}(x)^{2} \\
& \ldots \\
& T_{n}=T_{n}\left(F, \lambda_{0}, \lambda_{1}, \ldots, \lambda_{n-1}\right): \quad F_{n}(x)=\left(1+\lambda_{n-1}\right) F_{n-1}(x)-\lambda_{n-1} F_{n-1}(x)^{2}
\end{aligned}
$$

where $\left|\lambda_{i}\right| \leq 1, \forall i \in \overline{0, n-1}$ are the transmutation parameters.

We denote by $T_{1}\left(F, \lambda_{0}\right)$ the random variable with $F_{1}$ as its cumulative distribution function (cdf) and parameter $\lambda_{0}$, by $T_{2}\left(F, \lambda_{0}, \lambda_{1}\right)$ the random variable with $F_{2}$ as its cdf and parameters $\lambda_{0}, \lambda_{1}$, and so on, denoting by $T_{n}\left(F, \lambda_{0}, \lambda_{1}, \ldots, \lambda_{n-1}\right)$ the random variable with $F_{n}$ as its cdf and parameters $\lambda_{0}, \lambda_{1}, \cdots, \lambda_{n-1} . F_{n}$ is obtained by applying the transmutation map (1) to the previous cdf obtained at step $n-1, F_{n-1}$. Therefore, the construction of the $T_{n}$ models is recursive. 


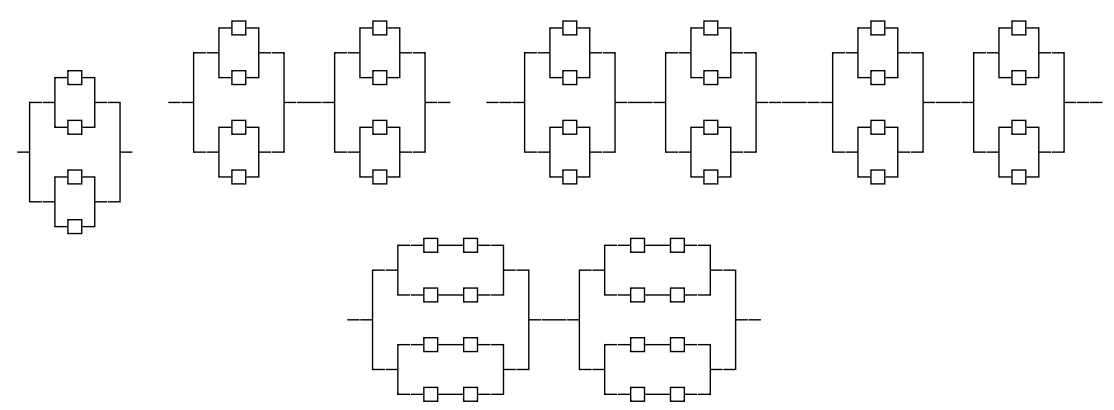

Figure 1: $T_{2}(F, 1,1), T_{3}(F, 1,1,-1), T_{4}(F, 1,1,-1,-1)$ and $T_{4}(F,-1,1,1,-1)$

\subsection{Motivation and interpretation}

The $T_{n}$ models can be interpreted as the lifetime distributions of some particular series-parallel/parallel-series system with independent and identically distributed components. A parallel system with $m$ components is a system that fails if all the components fail, while a series system with $m$ components is a system that fails if one of the components fails.

The cumulative distribution function $F_{n}$ for $\lambda_{i}=-1, \forall i=\overline{0, n-1}$ can be interpreted as the lifetime distribution function of a series system with $2^{n}$ independent identically distributed components which follow a common cdf $F$. For $\lambda_{i}=1, \forall i=\overline{0, n-1}, F_{n}$ can be interpreted as the lifetime distribution function of a parallel system with $2^{n}$ independent identically distributed components which follow a common $\operatorname{cdf} F$. When the transmutation parameters take different values, either -1 or $1, F_{n}$ can be interpreted as a more complex system.

The series-parallel/parallel-series systems that can be represented by the $T_{n}$ class of distributions are quiet complex for large values of $n$. They build up starting from either a parallel system with two components $\left(\lambda_{0}=1\right)$ or either a series system with two components $\left(\lambda_{0}=-1\right)$. At step $n$, the resulted system from the step $n-1$ is either put into a parallel system with two components $\left(\lambda_{n-1}=1\right)$, each component representing the system from the previous step, or into a series system with also two components $\left(\lambda_{n-1}=-1\right)$, each component representing the system from the previous step $n-1$. At step $n=1$ we can represent two types of systems, the parallel and series systems with two components, at step $n=2$ four series-parallel/parallel-series systems, while at step $n$ we can represent using the $T_{n}$ class of distributions, $2^{n}$ series-parallel/parallel-series systems. As examples, in Figures 1 and 2, we have displayed some possible types of systems that can be represented by the transmuted distributions of order $n$. The empty square represents a system's 


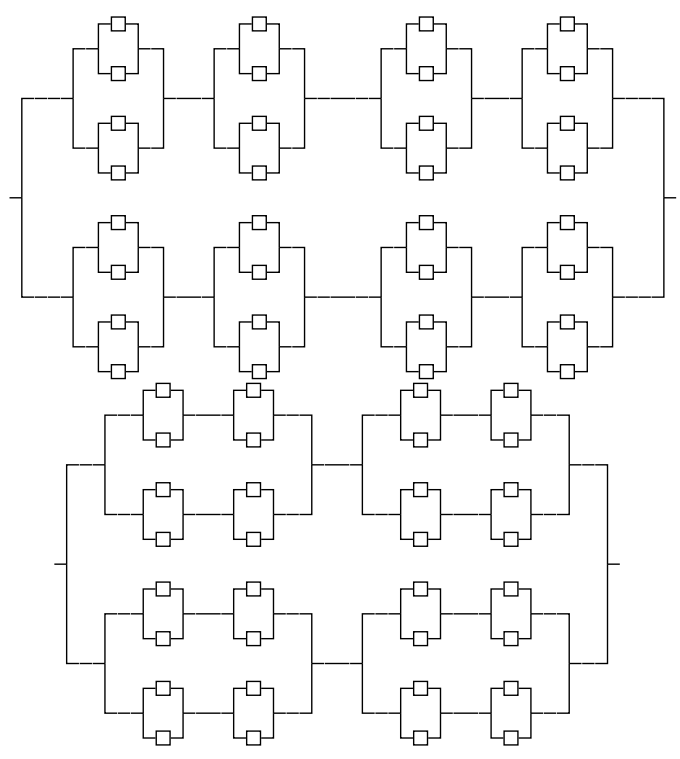

Figure 2: $T_{5}(F, 1,1,-1,-1,1)$ and $T_{5}(F, 1,-1,1,-1,1)$

component that has $F$ as its cumulative distribution function.

\subsection{Quantile function}

For generating values that follow a transmuted distribution of order $n$, we use the quantile function. Because the construction of the $T_{n}$ models is recursive, the generating of data algorithm is also recursive, but easy to implementate in software as $R$ or Matlab. We use this algorithm in section 4 for comparing expected system lifetimes of two series-parallel/parallel-series systems.

For $\lambda_{i} \neq 0$, the quantile function of $F_{n}$, denoted by $Q_{F_{n}}$, is defined as

$$
Q_{F_{n}}(u)=Q_{F_{n-1}}\left(u_{n-1}\right)=Q_{F_{n-2}}\left(u_{n-2}\right)=\cdots=Q_{F}\left(u_{0}\right), \quad u \in(0,1)
$$

where

$$
\begin{gathered}
u_{n-1}=\frac{1+\lambda_{n-1}-\sqrt{\left(1+\lambda_{n-1}\right)^{2}-4 \lambda_{n-1} u}}{2 \lambda_{n-1}}, \\
u_{n-2}=\frac{1+\lambda_{n-2}-\sqrt{\left(1+\lambda_{n-2}\right)^{2}-4 \lambda_{n-2} u_{n-1}}}{2 \lambda_{n-2}}
\end{gathered}
$$




$$
u_{0}=\frac{1+\lambda_{0}-\sqrt{\left(1+\lambda_{0}\right)^{2}-4 \lambda_{0} u_{1}}}{2 \lambda_{0}}
$$

and $Q_{F}$ is the quantile function of $F$.

For generating $m$ values for the $T_{n}$ class of distributions we have the following simple algorithm.

\section{Generating algorithm}

For every $j=\overline{1, m}$ we have the following steps

1. Generate $u$ from a Uniform $(0,1)$ distribution

2. Calculate $u_{n-1}, u_{n-2}, \ldots, u_{0}$

3. Set $x_{j}=Q_{F}\left(u_{0}\right)$.

\section{Characteristics of the $T_{n}$ class of distributions}

The aging process of a system is represented by its hazard and mean rezidual life functions. Using these two notions, we can determine in a unique way the distribution function of a random variable. In this section, we discuss the reliability, hazard rate and mean residual life functions of the $T_{n}$ models. Also, we discuss the order statistics of the $T_{n}$ class of distributions, focusing on the asymptotic behaviour of the extreme order statistics.

\subsection{Hazard rate, mean residual life and reliability functions}

There are three important functions used in reliability. These are the hazard rate, denoted by $h$, mean residual life, denoted by $M_{F}$, and reliability, denoted by $\bar{F}$, functions. Let $X$ be a random variable having $F$ as its cdf. The functions $h, M_{F}$ and $\bar{F}$ corresponding to $X$ are defined as follows

$$
\begin{aligned}
& h(x)=\frac{f(x)}{1-F(x)}, \quad M_{F}(x)=E(X-x \mid X \geq x)=\frac{1}{\bar{F}(x)} \int_{x}^{\infty} \bar{F}(t) d t \quad \text { and } \\
& \bar{F}(x)=1-F(x)=P(X<x) .
\end{aligned}
$$

The hazard rate function give us the probability of immediate failure of $X$, the mean residual life function, the remaining time of functioning after $X$ has survived $x$ years, while the reliability function give us the probability of surviving of $X$. 


\subsubsection{Reliability functions}

The reliability functions of the $T_{n}$ models are

$$
\begin{aligned}
& \bar{F}_{1}(x)=\left(1-\lambda_{0}\right) \bar{F}(x)+\lambda_{0} \bar{F}(x)^{2} \\
& \bar{F}_{2}(x)=\left(1-\lambda_{1}\right) \bar{F}_{1}(x)+\lambda_{1} \bar{F}_{1}(x)^{2} \\
& \bar{F}_{3}(x)=\left(1-\lambda_{2}\right) \bar{F}_{2}(x)+\lambda_{2} \bar{F}_{2}(x)^{2} \\
& \ldots \\
& \bar{F}_{n}(x)=\left(1-\lambda_{n-1}\right) \bar{F}_{n-1}(x)+\lambda_{n-1} \bar{F}_{n-1}(x)^{2}
\end{aligned}
$$

where $\bar{F}$ is the corresponding survival function of $F$.

\subsubsection{Hazard rate functions}

We denote by $h_{1}, h_{2}, \ldots, h_{n}$ the corresponding hazard rate functions of the transmutated distributions of order $n$, and by $h$ the hazard rate function of the baseline distribution $F$. The hazard rate function of the $T_{n}$ class of distributions can be represented as

$$
h_{n}(x)=h_{n-1}(x) \frac{1-\lambda_{n-1}+\lambda_{n-1} \bar{F}_{n-1}(x)}{1-\lambda_{n-1}+2 \lambda_{n-1} \bar{F}_{n-1}(x)}
$$

For $\lambda_{n-1}=1$ (parallel system), we have that the hazard rate function of the $T_{n}$ models is

$$
h_{n}(x)=\frac{1}{2} h_{n-1}(x)
$$

while for $\lambda_{n-1}=-1$ (series system), we have

$$
h_{n}(x)=h_{n-1}(x)\left(1+\frac{\bar{F}_{n-1}(x)}{2 F_{n-1}(x)}\right)
$$

\subsubsection{Mean residual life function}

We denote by $M_{F_{1}}, M_{F_{2}}, \ldots, M_{F_{n}}$ the corresponding mean residual life functions of $F_{1}, F_{2}, \ldots, F_{n}$. The mean residual life function of $T_{n}$ class of distributions is defined as

$$
M_{F_{n}}(x)=\frac{1}{\bar{F}_{n}(x)} \int_{x}^{\infty} \bar{F}_{n}(t) d t
$$


Asadi and Bayranmoglu (2006) [5] have discussed the mean residual life function of a $k$-out-of- $m$ structure. They gave the formulae for the mean residual life function of a $k$-out-of- $m$ system. It is well-known that a parallel system is a 1-out-of- $m$ system, while a series system is a $m$-out-of- $m$ system.

Given a $k$-out-of- $m$ system with $m$ components independent identically distributed with common cumulative distribution function $F$, the mean residual life function, denoted by $M_{m}^{k}$ of this system is defined as

$$
M_{m}^{k}(t)=\sum_{s=0}^{k-1}\left(\begin{array}{c}
m \\
s
\end{array}\right) \int_{t}^{\infty}\left(\frac{\bar{F}(x)}{\bar{F}(t)}\right)^{m-s}\left(1-\frac{\bar{F}(x)}{\bar{F}(t)}\right)^{s} d x
$$

The mean residual life function of a parallel system with $m$ components is

$$
M_{m}^{1}(t)=\int_{t}^{\infty}\left(\frac{\bar{F}(x)}{\bar{F}(t)}\right)^{n} d x
$$

while the mean residual life function of a series system with $m$ components is

$$
M_{m}^{m}(t)=\sum_{s=0}^{m-1}\left(\begin{array}{c}
m \\
s
\end{array}\right) \int_{t}^{\infty}\left(\frac{\bar{F}(x)}{\bar{F}(t)}\right)^{m-s}\left(1-\frac{\bar{F}(x)}{\bar{F}(t)}\right)^{s} d x
$$

The mean residual life functions of the $T_{n}$ models can be derived using equations (15) and (16). We give the formulae for the mean residual life functions of the $T_{1}, T_{2}$ models and a more generalized formulae for $T_{n}$ models when $\lambda_{n-1}$ is either 1 or -1 .

The mean residual life function of $T_{n}$ models when $\lambda_{n-1}=1$, using (15), is defined as

$$
M_{F_{n}}(t)=\int_{t}^{\infty}\left(\frac{\bar{F}_{n-1}(x)}{\bar{F}_{n-1}(t)}\right)^{2} d x
$$

The mean residual life function of $T_{n}$ models when $\lambda_{n-1}=-1$. using (16), is defined as

$$
M_{F_{n}}(t)=2 \int_{t}^{\infty} \frac{\bar{F}_{n-1}(x)}{\bar{F}_{n-1}(t)} d x-\int_{t}^{\infty}\left(\frac{\bar{F}_{n-1}(x)}{\bar{F}_{n-1}(t)}\right)^{2} d x
$$

For the $T_{1}$ models, we have two systems with two components, namely the parallel and series systems. Their mean residual life functions are obtained by taking $m$ equals 2 in equations (15) and (16). Using the $T_{2}$ models, we can model four series-parallel/parallel-series systems as follows: $\left(S_{1}\right)$ 
$T_{2}(F,-1,-1) ;\left(S_{2}\right) T_{2}(F, 1,1) ;\left(S_{3}\right) T_{2}(F, 1,-1)$ and $\left(S_{4}\right) T_{2}(F,-1,1)$. We denote by $M_{S_{1}}, M_{S_{2}}, M_{S_{3}}$ and $M_{S_{4}}$, respectively, the mean residual life functions of systems $S_{1}, S_{2}, S_{3}$ and $S_{4}$.

The mean residual life function of system $S_{1}$ is

$$
\begin{aligned}
M_{S_{1}}(t) & =2 \int_{t}^{\infty} \frac{\bar{F}(x)[1+F(x)]\left[1+F(x)^{2}\right]}{\bar{F}(t)[1+F(t)]\left[1+F(t)^{2}\right]} d x \\
& -\int_{t}^{\infty}\left(\frac{\bar{F}(x)[1+F(x)]\left[1+F(x)^{2}\right]}{\bar{F}(t)[1+F(t)]\left[1+F(t)^{2}\right]}\right)^{2} d x
\end{aligned}
$$

The mean residual life function of system $S_{2}$ is

$$
M_{S_{2}}(t)=\int_{t}^{\infty}\left(\frac{\bar{F}(x)^{4}}{\bar{F}(t)^{4}}\right)^{2} d x
$$

The mean residual life function of system $S_{3}$ is

$$
M_{S_{3}}(t)=2 \int_{t}^{\infty} \frac{\bar{F}(x)^{2}\left[2-\bar{F}(x)^{2}\right]}{\bar{F}(t)^{2}\left[2-\bar{F}(t)^{2}\right]} d x-\int_{t}^{\infty}\left(\frac{\bar{F}(x)^{2}\left[2-\bar{F}(x)^{2}\right]}{\bar{F}(t)^{2}\left[2-\bar{F}(t)^{2}\right]}\right)^{2} d x
$$

The mean residual life function of system $S_{4}$ is

$$
M_{S_{4}}(t)=\int_{t}^{\infty}\left(\frac{\bar{F}(x)^{2}[1+F(x)]^{2}}{\bar{F}(t)^{2}[1+F(t)]^{2}}\right)^{2} d x
$$

\subsection{Characteristics of the limiting distributions}

In this subsection, we discuss the order statistics of the $T_{n}$ models and their asymptotic behaviour. We show that the asymptotic distributions of the order statistics do not depend on the transmutation parameters. Because the transmutation parameters give us the structure of the complex seriesparallel/parallel-series systems modeled by the $T_{n}$ models, the asymptotic distributions of order statistics of these systems do not depend on the structure of them.

\subsubsection{Order statistics}

Let $X_{1: m} \leq X_{2: m} \leq \cdots \leq X_{m: m}$ be the order statistics of $X_{1}, X_{2}, \cdots, X_{m}$ random variables independent identically distributed with common cdf $F_{n}$ of the form (3). The density function $f_{i: m}(x)$ of the $i$ th order statistics, $X_{i: m}$, is given by 


$$
f_{i: m}(x)=\frac{f_{n}(x)}{B(i, m-i+1)} F_{n}(x)^{i-1} \bar{F}_{n}(x)^{m-i}
$$

where $B(a, b)=\int_{0}^{1} t^{a-1}(1-t)^{b-1} d t, a>0, b>0$, is the beta function.

Because the construction of $F_{n}$ starts with a baseline continuos distribution function $F$, the analytical formula in terms of $F$, of the density function $f_{i: m}$ is complicated and intractable for further developments. In this section, we give some general theorems which can be used to obtain the aymptotic distributions of the extreme order statistics given by $X_{1: m}=\min \left(X_{1}, X_{2}, \ldots, X_{m}\right)$ and $X_{m: m}=\max \left(X_{1}, X_{2}, \ldots, X_{m}\right)$.

Let $\mathcal{C}$ be the class of continuous cumulative distribution functions. Let

$$
\begin{gathered}
\mathcal{C}_{1}=\left\{F \in \mathcal{C} \mid \lim _{t \rightarrow 0} \frac{F(t x)}{F(t)}<\infty, \forall x\right\}, \\
\mathcal{C}_{2}=\left\{F \in \mathcal{C} \mid \lim _{t \rightarrow \infty} \frac{1-F(t+x)}{1-F(t)}<\infty, \forall x\right\}
\end{gathered}
$$

and

$$
\mathcal{C}_{3}=\left\{F \in \mathcal{C} \mid \lim _{x \rightarrow \infty} \frac{\mathrm{d}}{\mathrm{d} x}\left(\frac{1}{h(x)}\right)=0\right\}
$$

The following three theorems we use to give the asymptotic distributions of the extreme order statistics.

Theorem 1. If $F \in \mathcal{C}_{1}$, then also $F_{n} \in \mathcal{C}_{1}$.

Proof. For $n=1$ we have

$$
\lim _{t \rightarrow 0} \frac{F_{1}(t x)}{F_{1}(t)}=\lim _{t \rightarrow 0} \frac{F(t x)\left[1+\lambda_{0}-\lambda_{0} F(t x)\right]}{F(t)\left[1+\lambda_{0}-\lambda_{0} F(t x)\right]}=l(x)<\infty,
$$

where $l(x)=\lim _{t \rightarrow 0} \frac{F(t x)}{F(t)}$.

Now, for $(n-1) \rightarrow n$, we have

$$
\lim _{t \rightarrow 0} \frac{F_{n}(t x)}{F_{n}(t)}=\lim _{t \rightarrow 0} \frac{F_{n-1}(t x)\left[1+\lambda_{n-1}-\lambda_{n-1} F_{n-1}(t x)\right]}{F_{n-1}(t)\left[1+\lambda_{n-1}-\lambda_{n-1} F_{n-1}(t)\right]}=l(x)<\infty .
$$

By mathematical induction, we conclude the proof.

Remark 1. We notice that the limit of $F_{n}$ from $\mathcal{C}_{1}$ is invariant to the transmutation parameters $\lambda_{i}, i=\overline{0, n-1}$. 
Theorem 2. If $F \in \mathcal{C}_{2}$, then also $F_{n} \in \mathcal{C}_{2}$.

Proof. For proof see Appendix A.

Remark 2. It is very important to remark that the limit $\lim _{t \rightarrow \infty} \frac{1-F_{n}(t+x)}{1-F_{n}(t)}=$ $l(x)$, where $l(x)=\lim _{t \rightarrow \infty} \frac{1-F(t+x)}{1-F(t)}$, is invariant to the transmutation parameters $\lambda_{i}$.

Theorem 3. If $F \in \mathcal{C}_{3}$, then also $F_{n} \in \mathcal{C}_{3}$.

Proof. For proof see Appendix A.

\subsubsection{Extreme order statistics}

In this section, we give the asymptotic distributions of the order statistics of the $T_{n}$ models.

Theorem 4. Let $X_{1: m}$ and $X_{m: m}$ be the minimum and maximum of a random sample $X_{1}, X_{2}, \ldots, X_{m}$ from $F_{n}$ defined for $x>0$. The following statements hold

1. If $F \in \mathcal{C}_{1}$ and $\lim _{t \rightarrow 0} \frac{F(t x)}{F(t)}=x^{\theta_{1}}$, for each $x>0, \theta_{1}>0$, then

$$
\lim _{m \rightarrow \infty} P\left\{\frac{X_{1: m}-a_{m}}{b_{m}} \leq x\right\}=1-\exp \left(-x^{\theta_{1}}\right), \quad x>0 .
$$

2. If $F \in \mathcal{C}_{2}$ and $\lim _{t \rightarrow \infty} \frac{1-F(t+x)}{1-F(t)}=\exp \left(-\theta_{2} x\right)$ for each $x>0, \theta_{2}>0$, then

$$
\lim _{m \rightarrow \infty} P\left\{a_{m}^{*}\left(X_{m: m}-b_{m}^{*}\right) \leq x\right\}=\exp \left(-\exp \left(-\theta_{2} x\right)\right) .
$$

3. If $F \in \mathcal{C}_{3}$, then

$$
\lim _{m \rightarrow \infty} P\left\{\frac{X_{m: m}-c_{m}}{d_{m}} \leq x\right\}=\exp (-\exp (-x)), \quad x>0
$$

where $a_{m}, b_{m}, a_{m}^{*}, b_{m}^{*}, c_{m}, d_{m}$ are normalizing constants [4]. 
Proof. For (i), we apply Theorem 1 and Theorem 8.3.3 from [4] and for (ii), Theorem 2 and Theorem 1.6.2 from [10].

The last part, (iii), follows from Theorem 3 and Theorem 8.3.3 from [4].

The form of the normalizing constants can be determined following the Corollary 1.6.3 from [10] and the results from [4].

Theorem 5. Let $X_{1: m} \leq X_{2: m} \leq \cdots \leq X_{m: m}$ be the order statistics of a random sample $X_{1}, X_{2}, \cdots X_{m}$ from $F_{n}$ defined for $x>0$. The following statements hold

1. If $F \in \mathcal{C}_{1}$ and $\lim _{t \rightarrow 0} \frac{F(t x)}{F(t)}=x^{\theta_{1}}$ for each $x>0, \theta_{1}>0$, then for each $i=\overline{1, m}$

$$
\lim _{m \rightarrow \infty} P\left\{\frac{X_{i: m}-a_{m}}{b_{m}} \leq x\right\}=1-\sum_{k=0}^{i-1} \frac{x^{k \theta_{1}}}{k !} \exp \left\{-x^{\theta_{1}}\right\}, \quad x>0
$$

2. If $F \in \mathcal{C}_{3}$, then for each $i=\overline{1, m}$

$$
\lim _{m \rightarrow \infty} P\left\{\frac{X_{m-i+1: m}-c_{m}}{d_{m}} \leq x\right\}=\sum_{r=0}^{i-1} \frac{\exp (-r x)}{r !} \exp (-\exp (-x))
$$

where $a_{m}, b_{m}, c_{m}$ and $d_{m}$ are normalizing constants [4].

Proof. The proof follows from Theorem 1, Theorem 3 and Eqs. (8.4.2) and (8.4.3) of [4].

\section{Stochastic ordering}

In this section, we discuss stochastic ordering, namely the likelihood ratio ordering of the transmuted distributions of order $n$ with applications in comparing the expected system lifetimes of complex series-parallel/parallel-series systems.

Definition 1. [17] Let $X_{1}$ and $X_{2}$ be two random variables with respective cumulative distribution functions $F_{1}$ and $F_{2}$ and hazard rates $h_{1}$ and $h_{2}$, respectively. Also, let $f_{1}$ and $f_{2}$ be the probability density functions corresponding to $F_{1}$ and $F_{2}$, respectively. 
1. $X_{1}$ is said to be smaller than $X_{2}$ in the likelihood ratio order (denoted by $\left.X_{1} \underset{L R}{\leq} X_{2}\right)$, if $\frac{f_{2}(x)}{f_{1}(x)}$ is non-decreasing over the union of the supports of $X_{1}$ and $X_{2}$,

2. $X_{1}$ is said to be stochastically smaller than $X_{2}$, denoted by $X_{1} \leq_{S T} X_{2}$, if $F_{1}(x) \geq F_{2}(x)$ for all $x$.

3. $X_{1}$ is said to be smaller than $X_{2}$ in the hazard rate order, denoted by $X_{1} \leq_{H R} X_{2}$, if $h_{1}(x) \leq h_{2}(x)$ for all $x$.

Remark 3. It is well-known that the likelihood ratio is stronger than the hazard rate order and the stochastic order, $X_{1} \underset{L R}{\leq} X_{2} \Rightarrow X_{1} \leq_{H R} X_{2} \Rightarrow$ $X_{1} \leq_{S T} X_{2}$. Also, we have that $X_{1} \leq_{S T} X_{2}$ implies $E\left(X_{1}\right) \leq E\left(X_{2}\right)$ [17].

\subsection{Stochastic ordering of the $T_{n}$ models}

Let $F$ and $G$ be two arbitrary continuous cumulative distribution functions and let

$$
\begin{aligned}
& F_{1}(x)=\left(1+\lambda_{0}\right) F(x)-\lambda_{0} F(x)^{2} \\
& F_{2}(x)=\left(1+\lambda_{1}\right) F_{1}(x)-\lambda_{1} F_{1}(x)^{2} \\
& F_{3}(x)=\left(1+\lambda_{2}\right) F_{2}(x)-\lambda_{2} F_{2}(x)^{2} \\
& \ldots \\
& F_{n}(x)=\left(1+\lambda_{n-1}\right) F_{n-1}(x)-\lambda_{n-1} F_{n-1}(x)^{2}
\end{aligned}
$$

and

$$
\begin{aligned}
& G_{1}(x)=\left(1+\lambda_{0}^{\prime}\right) G(x)-\lambda_{0}^{\prime} G(x)^{2} \\
& G_{2}(x)=\left(1+\lambda_{1}^{\prime}\right) G_{1}(x)-\lambda_{1}^{\prime} G_{1}(x)^{2} \\
& G_{3}(x)=\left(1+\lambda_{2}^{\prime}\right) G_{2}(x)-\lambda_{2}^{\prime} G_{2}(x)^{2} \\
& \ldots \\
& G_{n}(x)=\left(1+\lambda_{n-1}^{\prime}\right) G_{n-1}(x)-\lambda_{n-1}^{\prime} G_{n-1}(x)^{2} .
\end{aligned}
$$

We denote the corresponding probability density functions by $f_{1}, f_{2}, \ldots, f_{n}$ and $g_{1}, g_{2}, \ldots, g_{n}$, respectively. 
Theorem 6. Let $X, X_{1}, X_{2}, \ldots, X_{n}$ be random variables with cumulative distribution functions $F, F_{1}, F_{2}, \ldots, F_{n}$, respectively. Let $Y, Y_{1}, Y_{2}, \ldots, Y_{n}$ be random variables with cumulative distribution functions $G, G_{1}, G_{2}, \ldots, G_{n}$, respectively. If $-1 \leq \lambda_{i} \leq 0 \leq \lambda_{i}^{\prime} \leq 1$, for all $i=\overline{0, n-1}$ and $Y \underset{L R}{\leq} X$, then $Y_{i} \underset{L R}{\leq} X_{i}$ for all $i=\overline{1, n}$.

Proof. We have

$$
\begin{aligned}
& \frac{f_{i}(x)}{g_{i}(x)}=\frac{f_{i-1}(x)\left[1+\lambda_{i-1}-2 \lambda_{i-1} F_{i-1}(x)\right]}{g_{i-1}(x)\left[1+\lambda_{i-1}^{\prime}-2 \lambda_{i-1}^{\prime} G_{i-1}(x)\right]} \\
& =\frac{f_{i-2}(x)\left[1+\lambda_{i-2}-2 \lambda_{i-2} F_{i-2}(x)\right]}{g_{i-2}(x)\left[1+\lambda_{i-2}^{\prime}-2 \lambda_{i-2}^{\prime} G_{i-2}(x)\right]} \frac{\left[1+\lambda_{i-1}-2 \lambda_{i-1} F_{i-1}(x)\right]}{\left[1+\lambda_{i-1}^{\prime}-2 \lambda_{i-1}^{\prime} G_{i-1}(x)\right]}=\cdots= \\
& =\frac{f(x)}{g(x)} \frac{\left[1+\lambda_{0}-2 \lambda_{0} F(x)\right]\left[1+\lambda_{1}-2 \lambda_{1} F_{1}(x)\right] \cdots\left[1+\lambda_{i-1}-2 \lambda_{i-1} F_{i-1}(x)\right]}{\left[1+\lambda_{0}^{\prime}-2 \lambda_{0}^{\prime} G(x)\right]\left[1+\lambda_{1}^{\prime}-2 \lambda_{1}^{\prime} G_{1}(x)\right] \cdots\left[1+\lambda_{i-1}^{\prime}-2 \lambda_{i-1}^{\prime} G_{i-1}(x)\right]}
\end{aligned}
$$

and

$$
\begin{aligned}
\log \frac{f_{i}(x)}{g_{i}(x)} & =\log \frac{f(x)}{g(x)}+\log \left[1+\lambda_{0}-2 \lambda_{0} F(x)\right]-\log \left[1+\lambda_{0}^{\prime}-2 \lambda_{0}^{\prime} G(x)\right] \\
& +\log \left[1+\lambda_{1}-2 \lambda_{1} F_{1}(x)\right]-\log \left[1+\lambda_{1}^{\prime}-2 \lambda_{1}^{\prime} G_{1}(x)\right] \\
& +\cdots+\log \left[1+\lambda_{i-1}-2 \lambda_{i-1} F_{i-1}(x)\right]-\log \left[1+\lambda_{i-1}^{\prime}-2 \lambda_{i-1}^{\prime} G_{i-1}(x)\right]
\end{aligned}
$$

$\frac{f_{i}(x)}{g_{i}(x)}$ is non-decreasing if and only if $\left(\log \frac{f_{i}(x)}{g_{i}(x)}\right)^{\prime} \geq 0$ for all $x$, where

$$
\begin{aligned}
\left(\log \frac{f_{i}(x)}{g_{i}(x)}\right)^{\prime} & =\left(\log \frac{f(x)}{g(x)}\right)^{\prime}-\frac{2 \lambda_{0} f(x)}{1+\lambda_{0}-2 \lambda_{0} F(x)}+\frac{2 \lambda_{0}^{\prime} g(x)}{1+\lambda_{0}^{\prime}-2 \lambda_{0}^{\prime} G(x)} \\
& -\frac{2 \lambda_{1} f_{1}(x)}{1+\lambda_{1}-2 \lambda_{1} F_{1}(x)}+\frac{2 \lambda_{1}^{\prime} g_{1}(x)}{1+\lambda_{1}^{\prime}-2 \lambda_{1}^{\prime} G_{1}(x)} \\
& -\cdots-\frac{2 \lambda_{i-1} f_{i-1}(x)}{1+\lambda_{i-1}-2 \lambda_{i-1} F_{i-1}(x)}+\frac{2 \lambda_{i-1}^{\prime} g_{i-1}(x)}{1+\lambda_{i-1}^{\prime}-2 \lambda_{i-1}^{\prime} G_{i-1}(x)}
\end{aligned}
$$

It is easy to see that $-1 \leq \lambda_{i} \leq 0 \leq \lambda_{i}^{\prime} \leq 1$ for all $i=\overline{0, n-1}$ and $Y \underset{L R}{\leq} X$ imply $\left(\log \frac{f_{i}(x)}{g_{i}(x)}\right)^{\prime} \geq 0$ for all $x$, and the result holds.

The theorem above generalizes a proven statament that tells us that a parallel system is always preferable than a series system [14]. 

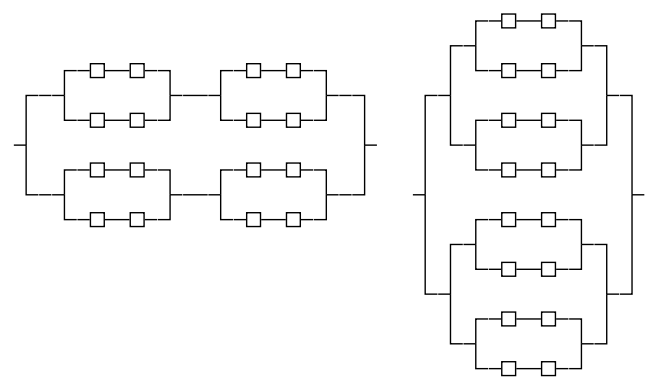

Figure 3: The serie-parallel/parallel-series systems represented by $X$ and $Y$.

Remark 4. In Theorem 6 if $\lambda_{n-1} \leq \lambda_{n-1}^{\prime}$ and $F_{n-1}=G_{n-1}$, then $Y_{n} \underset{L R}{\leq}$ $X_{n}$.

\subsection{Comparing series-parallel/parallel-series systems}

Let $F(x)=1-\exp \left\{-(x / \rho)^{\mu}\right\}, x>0$ be a Weibull cumulative distribution function of parameters $\mu>0$ and $\rho>0$. Let $X$ and $Y$ be random variables having $F_{4}$ and $G_{4}$ as their cumulative distribution functions obtained using the method described in Section 1 as follows: $F_{4}$ is the cumulative distribution function of $T_{4}(F,-1,1,-1,1)$, while $G_{4}$ is the cumulative distribution function of $T_{4}(F,-1,1,1,1)$. In Figure 3, we have displayed the series-parallel/parallelseries systems that these random variables $X$ and $Y$ represent.

Using Theorem 6 and Remarks 3 and 4, we have $Y \underset{L R}{\leq} X$, and therefore, the expected system lifetime of $Y$ is smaller than the expected system lifetime of $X$. Generating values from $X$ and $Y$ using the algorithm presented in subsection 2.2, we have calculated the expected system lifetime of them. These values are displayed in Table 1 .

\begin{tabular}{|c|c|c|c|c|c|}
\hline$(\mu, \rho)$ & $E(X)$ & $E(Y)$ & $(\mu, \rho)$ & $E(X)$ & $E(Y)$ \\
\hline$(2,4)$ & 3.701494 & 2.358217 & $(0.7,0.5)$ & 0.4525109 & 0.1389469 \\
$(0.7,1)$ & 0.9122977 & 0.2844805 & $(1,0.9)$ & 0.8163832 & 0.3459043 \\
$(3,0.8)$ & 0.7531299 & 0.5558009 & $(7,1)$ & 0.971466 & 0.8491724 \\
$(4,7)$ & 6.688466 & 5.285205 & $(5,5)$ & 4.812751 & 3.988665 \\
$(3,2)$ & 1.886318 & 1.389314 & $(10,20)$ & 19.60123 & 17.82688 \\
\hline
\end{tabular}

Table 1: The expected system lifetimes of $X$ and $Y$. 


\section{$5 \quad$ Parameter estimation}

Let $x_{1}, x_{2}, \ldots, x_{m}$ be a random sample of size $m$ from $T_{n}\left(F, \lambda_{0}, \lambda_{1}, \cdots, \lambda_{n-1}\right)$ of parameters $\left(\theta, \Lambda_{t}\right)$, where $\theta=\left(\theta_{1}, \theta_{2}, \cdots \theta_{k}\right)$ is the vector parameter of length $k$ of the underlying distribution $F$ and $\Lambda_{t}=\left(\lambda_{0}, \lambda_{1}, \ldots, \lambda_{t}\right)$ are the transmutation parameters, $t=\overline{0, n-1}$. The method used to estimate the parameters is the maximum likelihood estimation.

The log-likelihood function is given by

$$
\mathcal{L}=\sum_{i=1}^{m}\left\{\log \left[f_{n-1}\left(x_{i} ; \theta, \Lambda_{n-2}\right)\right]+\log \left[1+\lambda_{n-1}-2 \lambda_{n-1} F_{n-1}\left(x_{i} ; \theta, \Lambda_{n-2}\right)\right]\right\}
$$

In order to maximize the log-likelihood function, we solve the nonlinear likelihood system obtained by differentiating (27). This can be done using $R$, Matlab and Mathcad, among other packages. The elements of the score vector $V\left(\rho_{t}\right), \rho_{t}=\left(\theta, \Lambda_{t}\right), \Lambda_{t}=\left(\lambda_{0}, \lambda_{1}, \ldots, \lambda_{t}\right)$ and $\theta=\left(\theta_{1}, \theta_{2}, \ldots, \theta_{k}\right)$ are

$V_{\theta_{s}}=\sum_{i=1}^{m}\left\{\frac{\frac{\partial}{\partial \theta_{s}} f_{n-1}\left(x_{i} ; \rho_{n-2}\right)}{f_{n-1}\left(x_{i} ; \rho_{n-2}\right)}-\frac{2 \lambda_{n-1} \frac{\partial}{\partial \theta_{s}} F_{n-1}\left(x_{i} ; \rho_{n-2}\right)}{1+\lambda_{n-1}-2 \lambda_{n-1} F_{n-1}\left(x_{i} ; \rho_{n-2}\right)}\right\}, \quad s=\overline{1, k}$

where

$$
\begin{aligned}
\frac{\partial}{\partial \theta_{s}} f_{n-1}\left(x_{i} ; \rho_{n-2}\right) & =\frac{\partial}{\partial \theta_{s}} f_{n-2}\left(x_{i} ; \rho_{n-3}\right)\left[1+\lambda_{n-2}-2 \lambda_{n-2} F_{n-2}\left(x_{i} ; \rho_{n-3}\right)\right. \\
& \left.-2 \lambda_{n-2} f_{n-2}\left(x_{i} ; \rho_{n-3}\right) \frac{\partial}{\partial \theta_{s}} F_{n-2}\left(x_{i} ; \rho_{n-3}\right)\right] \\
\frac{\partial}{\partial \theta_{s}} F_{n-2}\left(x_{i} ; \rho_{n-3}\right) & =\frac{\partial}{\partial \theta_{s}} F_{n-3}\left(x_{i} ; \rho_{n-3}\right)\left[1+\lambda_{n-3}-2 \lambda_{n-3} F_{n-3}\left(x_{i} ; \rho_{n-3}\right)\right]
\end{aligned}
$$

and

$$
\begin{aligned}
V_{\lambda_{j}} & =\sum_{i=1}^{m}\left\{\frac{\frac{\partial}{\partial \lambda_{j}} f_{n-1}\left(x_{i} ; \rho_{n-2}\right)}{f_{n-1}\left(x_{i} ; \rho_{n-2}\right)}-\frac{2 \lambda_{n-1} \frac{\partial}{\partial \lambda_{j}} F_{n-1}\left(x_{i} ; \rho_{n-2}\right)}{1+\lambda_{n-1}-2 \lambda_{n-1} F_{n-1}\left(x_{i} ; \rho_{n-2}\right)}\right\} \\
j & =\overline{0, n-2}
\end{aligned}
$$

and 


$$
V_{\lambda_{n-1}}=\sum_{i=1}^{m} \frac{1-2 F_{n-1}\left(x_{i} ; \rho_{n-2}\right)}{1+\lambda_{n-1}-2 \lambda_{n-1} F_{n-1}\left(x_{i} ; \rho_{n-2}\right)}
$$

where

$$
\begin{aligned}
& \frac{\partial}{\partial \lambda_{j}} f_{n}\left(x_{i} ; \rho_{n-1}\right)=\left[1+\lambda_{n-1}-2 \lambda_{n-1} F_{n-1}\left(x_{i} ; \rho_{n-2}\right)\right] \frac{\partial}{\partial \lambda_{j}} f_{n-1}\left(x_{i} ; \rho_{n-2}\right) \\
&-2 \lambda_{n-1} f_{n-1}\left(x_{i} ; \rho_{n-2}\right) \frac{\partial}{\partial \lambda_{j}} F_{n-1}\left(x_{i} ; \rho_{n-2}\right) \\
& \frac{\partial}{\partial \lambda_{j}} F_{n}\left(x_{i} ; \rho_{n-1}\right)=\left[1+\lambda_{n-1}-2 \lambda_{n-1} F_{n-1}\left(x_{i} ; \rho_{n-2}\right)\right] \frac{\partial}{\partial \lambda_{j}} F_{n-1}\left(x_{i} ; \rho_{n-2}\right), \\
& j \neq n-1
\end{aligned}
$$

Because we can have a relatively large number of parameters this can cause problems especially when the sample size is not large. A good set of initial values is essential. The second partial derivatives of the log-likelihood function for the construction of the Fisher information matrix are also obtained. These are presented in the Appendix B.

\section{Goodness-of-fit}

In this section, using two data sets we compare the fits of the $T_{n}$ distributions considering as baseline distribution functions the exponentiated power Lindley (EPL) [6] and Pareto [12] distributions. In each case the parameters are estimated by maximum likelihood using fitdist() function in $R$ with Nelder-Mead options used as an iterative process for maximizing the log-likelihood function. First we give the MLEs of the parameters and the values of the Akaike Information Criterion (AIC) and Bayesian Information Criterion (BIC) statistics. The lower the values of these criteria the better the fit. Next we perform Kolmogorov-Smirnov tests. The Kolmogorov-Smirnov test is performed using the ks.test() function in $R$. Finally, we provide the empirical and teoretical cdf for data set 1 to show a visual comparison of the fitted cdf functions. Also, we consider the TTT-plot transformation which gives us the shape of the hazard rate function [1]. If the TTT curve is convex, then the hazard rate is increasing, if it is concave, then the hazard rate is decreasing, if it is convex then concave, then it is upside-down shaped, otherwise it is bathtub shaped [1]. The data sets are as follows. 


\begin{tabular}{|c|c|c|c|c|c|}
\hline & Log-lik & AIC & BIC & K-S & p-value \\
\hline$T_{5}\left(P L, \lambda_{0}\right)$ & -120.8433 & 247.6866 & 254.6788 & 0.078578 & 0.7061 \\
$T_{5}\left(E P L, \lambda_{0}\right)$ & -120.5105 & 249.0209 & 258.3438 & 0.080024 & 0.6849 \\
$T_{1}\left(E L, \lambda_{0}\right)$ & -121.2876 & 248.5752 & 255.5674 & 0.08935983 & 0.5485178 \\
$P L$ & -122.4001 & 248.8001 & 253.4616 & 0.1123079 & 0.2721916 \\
EPL & -121.8663 & 249.7326 & 256.7248 & 0.09929166 & 0.4150025 \\
$T_{1}\left(E P L, \lambda_{0}\right)$ & -121.2683 & 250.5366 & 259.8596 & 0.09099421 & 0.5253884 \\
$T_{7}\left(E P L, \lambda_{0}\right)$ & -120.3936 & 248.7873 & 258.1102 & 0.07837921 & 0.7090126 \\
\hline
\end{tabular}

Table 2: The criteria information for data set 1 .

\section{Data Set 1}

The first data set [3] we consider is the times of fatigue fracture of Kevlar 373/epoxy that are subject to constant pressure at $90 \%$ stress level until all have failed: $x_{1}=(0.0251,0.0886,0.0891,0.2501,0.3113,0.3451,0.4763$, $0.5650,0.5671,0.6566,0.6748,0.6751,0.6753,0.7696,0.8375,0.8391,0.8425$, $0.8645,0.8851,0.9113,0.9120,0.9836,1.0483,1.0596,1.0773,1.1733,1.2570$, $1.2766,1.2985,1.3211,1.3503,1.3551,1.4595,1.4880,1.5728,1.5733,1.7083$, $1.7263,1.7460,1.7630,1.7746,1.8275,1.8375,1.8503,1.8808,1.8878,1.8881$, $1.9316,1.9558,2.0048,2.0408,2.0903,2.1093,2.1330,2.2100,2.2460,2.2878$, 2.3203, 2.3470, 2.3513, 2.4951, 2.5260, 2.9911, 3.0256, 3.2678, 3.4045, 3.4846, $3.7433,3.7455,3.9143,4.8073,5.4005,5.4435,5.5295,6.5541,9.0960)$. This data set has an upside-down hazard rate function, see Figures 4 and 5.

\section{Data Set 2}

Merovci and Puka (2014) [12] used two data sets for the fitting of the transmuted Pareto distribution, $X \sim \operatorname{Pareto}\left(x_{0}, a\right), a>0$ and $x_{0}$ the necessarily positive minimum possible value of $X$ and compared it to Pareto, generalized Pareto and exponentiated Weibull distributions. They showed that the transmuted Pareto distribution is a better fit model in both cases. Using one of the two data sets from [12], we extend the data analysis study, fitting the transmuted Pareto of order $2\left(T_{2}\right.$ (Pareto, $\left.\left.\lambda_{0}, \lambda_{1}\right)\right)$ and transmuted Pareto of order $3\left(T_{3}\left(\right.\right.$ Pareto, $\left.\left.\lambda_{0}, \lambda_{1}, \lambda_{2}\right)\right)$ distributions to this choosen data set. The data set corresponds to the Floyd River located in James, Iowa, USA.

For data set 1 we consider the fittings of the transmutated power Lindley of order 5 distribution $\left(T_{5}\left(P L, \lambda_{0}\right)\right)$ with $\lambda_{0}=\lambda_{1}=\lambda_{2}=\lambda_{3}=\lambda_{4}$, transmuted exponentiated power Lindley of order 5 distribution $\left(T_{5}\left(E P L, \lambda_{0}\right)\right)$ with $\lambda_{0}=\lambda_{1}$ $=\lambda_{2}=\lambda_{3}=\lambda_{4}$, transmuted exponentiated Lindley distribution $\left(T_{1}\left(E P, \lambda_{0}\right)\right)$, power Lindley distribution $(P L)$, exponentiated power Lindley distribution 

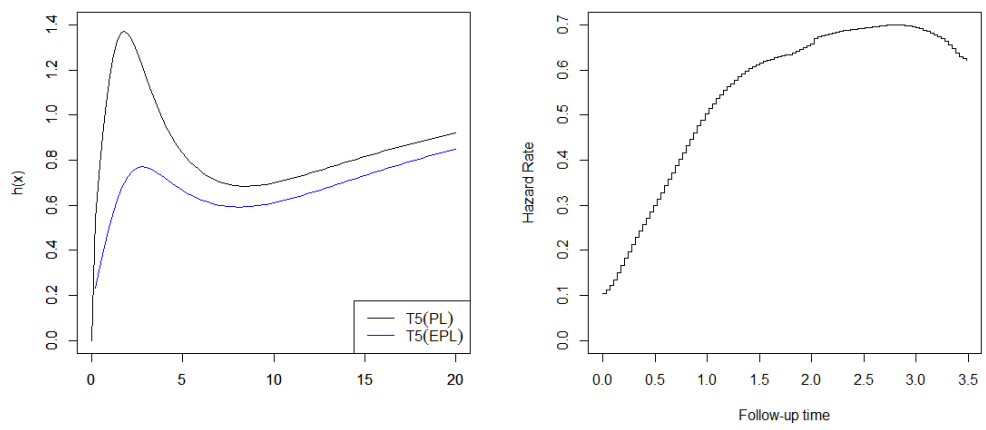

Figure 4: Hazard rate function for example 1.

\begin{tabular}{|c|c|c|c|c|}
\hline & $\hat{\beta}$ & $\hat{\alpha}$ & $\hat{\theta}$ & $\hat{\lambda_{0}}$ \\
\hline$T_{5}\left(P L, \lambda_{0}\right)$ & 1.3342506 & - & 0.2709258 & 0.6203250 \\
$T_{5}\left(E P L, \lambda_{0}\right)$ & 1.4128086 & 0.9035090 & 0.1880978 & 0.4456854 \\
$T_{1}\left(E L, \lambda_{0}\right)$ & - & 1.4145876 & 0.7177399 & 0.7058371 \\
$P L$ & 1.1422729 & - & 0.7047831 & - \\
$E P L$ & 0.9499608 & 1.5355626 & 1.0205424 & - \\
$T_{1}\left(E P L, \lambda_{0}\right)$ & 1.0493532 & 1.3046813 & 0.6640713 & 0.6889066 \\
$T_{7}\left(E P L, \lambda_{0}\right)$ & 1.5596811 & 0.8153664 & 0.1200829 & 0.3746730 \\
\hline
\end{tabular}

Table 3: The MLE's for data set 1.

$(E P L)$, transmuted exponentiated power Lindley distribution $\left(T_{1}\left(E P L, \lambda_{0}\right)\right)$ and transmuted exponentiated power Lindley of order 7 distribution $\left(T_{7}(E P L\right.$, $\left.\lambda_{0}\right)$ ) with $\lambda_{0}=\lambda_{1}=\lambda_{2}=\lambda_{3}=\lambda_{4}=\lambda_{5}=\lambda_{6}$. In Table 2 , the values coorresponding to the information criteria considered, AIC and BIC, along with the p-values of the Kolmogorov-Smirnov tests are displayed. The values of MLE estimators are presented in Table 3. As it can be noticed, the $T_{5}\left(P L, \lambda_{0}\right)$ distribution has the lowest AIC and BIC. In Figure 5, we have plotted the TTT plot transformation.

For data set 2, the transmuted Pareto of order 3 distribution has the lowest AIC and BIC. The results are displayed in Table 4. Also, for this data set, we consider hypothesis tests. We compare the $T_{3}$ (Pareto, $\left.\lambda_{0}, \lambda_{1}, \lambda_{2}\right)$ model with $T_{2}\left(\right.$ Pareto, $\left.\lambda_{0}, \lambda_{1}\right)$ and $T_{1}$ (Pareto, $\left.\lambda_{0}\right)$ models. The results are displayed in Table 5 . 

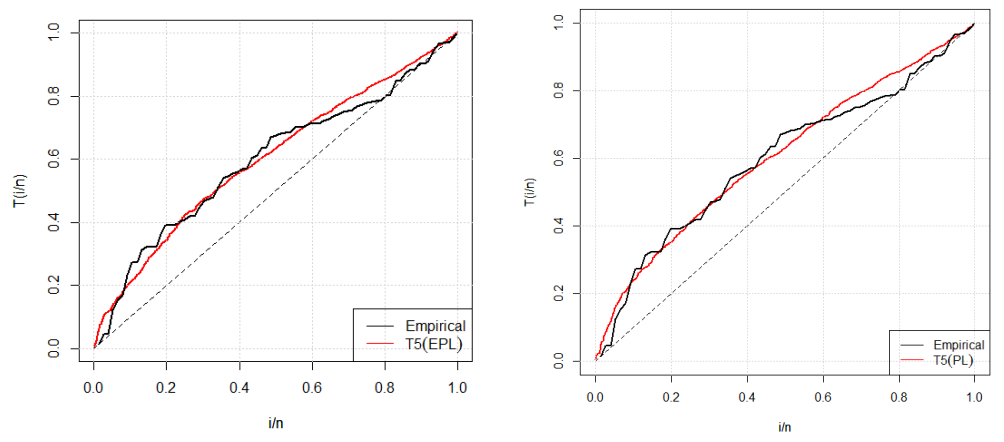

Figure 5: The TTT-transform plot for example 1.

\begin{tabular}{|c|c|c|c|c|}
\hline & $\hat{a}$ & $\hat{\lambda}_{0}$ & $\hat{\lambda}_{1}$ & $\hat{\lambda}_{2}$ \\
\hline$T_{3}\left(\right.$ Pareto, $\left.\lambda_{0}, \lambda_{1}, \lambda_{2}\right)$ & 0.9456290 & -0.7394268 & -0.7784058 & -0.8363968 \\
$T_{2}\left(\right.$ Pareto $\left., \lambda_{0}, \lambda_{1}\right)$ & 0.7755660 & -0.8469511 & -0.8843003 & - \\
$T_{1}$ (Pareto, $\left.\lambda_{0}\right)$ & 0.5857841 & -0.9103600 & - & - \\
\hline
\end{tabular}

Table 4: The MLE's for data set 2.

\section{Appendix A}

Proof of Theorem 2

Proof. For $n=1$, we have

$$
\begin{aligned}
& \lim _{t \rightarrow \infty} \frac{\bar{F}_{1}(t+x)}{\bar{F}_{1}(t)}=\lim _{t \rightarrow \infty} \frac{\bar{F}(t+x)\left[1-\lambda_{0}+\lambda_{0} \bar{F}(t+x)\right]}{\bar{F}(t)\left[1-\lambda_{0}+\lambda_{0} \bar{F}(t)\right]}=l(x)<\infty, \forall x, \\
& \text { where } l(x)=\lim _{t \rightarrow \infty} \frac{\bar{F}(t+x)}{\bar{F}(t)}
\end{aligned}
$$

\begin{tabular}{|c|c|c|c|c|}
\hline & AIC & BIC & K-S & p-value \\
\hline$T_{3}\left(\right.$ Pareto, $\left.\lambda_{0}, \lambda_{1}, \lambda_{2}\right)$ & 764.6231 & 771.2773 & 0.10025 & 0.7914 \\
$T_{2}\left(\right.$ Pareto, $\left.\lambda_{0}, \lambda_{1}\right)$ & 767.3783 & 772.369 & 0.15253 & 0.2936 \\
$T_{1}\left(\right.$ Pareto,$\left.\lambda_{0}\right)$ & 774.6983 & 778.0254 & 0.235 & 0.02232 \\
\hline
\end{tabular}

Table 5: The criteria comparison for data set 2 . 


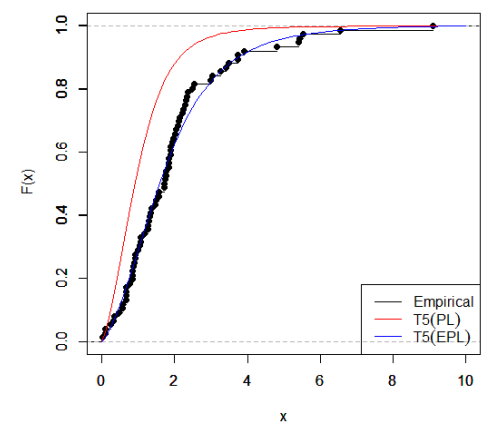

Figure 6: The empirical cdf for example 1.

\begin{tabular}{|c|c|c|}
\hline & $\mathrm{w}$ & $\mathrm{p}$-value \\
\hline$T_{3}\left(\right.$ Pareto $\left., \lambda_{0}, \lambda_{1}, \lambda_{2}\right)$ vs $T_{2}\left(\right.$ Pareto $\left., \lambda_{0}, \lambda_{1}\right)$ & 7.658383 & 0.005650913 \\
$T_{3}\left(\right.$ Pareto, $\left.\lambda_{0}, \lambda_{1}, \lambda_{2}\right)$ vs $T_{1}\left(\right.$ Pareto,$\left.\lambda_{0}\right)$ & 12.56532 & 0.001868428 \\
\hline
\end{tabular}

Table 6: Comparison of submodels for data set 2

Now, for $(n-1) \rightarrow n$, we have

$$
\lim _{t \rightarrow \infty} \frac{\bar{F}_{n}(t+x)}{\bar{F}_{n}(t)}=\lim _{t \rightarrow \infty} \frac{\bar{F}_{n-1}(t+x)\left[1-\lambda_{n-1}+\lambda_{n-1} \bar{F}_{n-1}(t+x)\right]}{\bar{F}_{n-1}(t)\left[1-\lambda_{n-1}+\lambda_{n-1} \bar{F}_{n-1}(t)\right]}=l(x) .
$$

By mathematical induction, we conclude the proof.

\section{Proof of Theorem 3}

Proof. Rewriting the corresponding density functions of $F_{1}, F_{2}, \cdots, F_{n}$ in terms of survival functions, we get

$$
\begin{aligned}
& f_{1}(x)=f(x)\left[1-\lambda_{0}+2 \lambda_{0} \bar{F}(x)\right] \\
& f_{2}(x)=f_{1}(x)\left[1-\lambda_{1}+2 \lambda_{1} \bar{F}_{1}(x)\right] \\
& f_{3}(x)=f_{2}(x)\left[1-\lambda_{2}+2 \lambda_{2} \bar{F}_{2}(x)\right] \\
& \ldots \\
& f_{n}(x)=f_{n-1}(x)\left[1-\lambda_{n-1}+2 \lambda_{n-1} \bar{F}_{n-1}(x)\right]
\end{aligned}
$$


For $n=1$, we have

$$
\begin{aligned}
\frac{d}{d x} \frac{1}{h_{1}(x)} & =\frac{d}{d x} \frac{\bar{F}_{1}}{f_{1}(x)}=\frac{d}{d x} \frac{\bar{F}(x)\left[1-\lambda_{0}+\lambda_{0} \bar{F}(x)\right]}{f(x)\left[1-\lambda_{0}+2 \lambda_{0} \bar{F}(x)\right]} \\
& =-\left(\frac{d}{d x} \frac{\bar{F}(x)}{f(x)}\right) \frac{1-\lambda_{0}+\lambda_{0} \bar{F}(x)}{1-\lambda_{0}+2 \lambda_{0} \bar{F}(x)}+\frac{\lambda_{0} \bar{F}(x)\left[\lambda_{0}-1\right]}{\left[1-\lambda_{0}+2 \lambda_{0} \bar{F}(x)\right]^{2}}
\end{aligned}
$$

Because $F \in \mathcal{C}_{3}$, we have

$$
\lim _{x \rightarrow \infty} \frac{1-\lambda_{0}+\lambda_{0} \bar{F}(x)}{1-\lambda_{0}+2 \lambda_{0} \bar{F}(x)}=1 \quad \text { and } \quad \lim _{x \rightarrow \infty} \frac{\lambda_{0} \bar{F}(x)\left[\lambda_{0}-1\right]}{\left[1-\lambda_{0}+2 \lambda_{0} \bar{F}(x)\right]^{2}}=0
$$

Therefore $\lim _{x \rightarrow \infty} \frac{d}{d x} \frac{1}{h_{1}(x)}=0$.

Now, for $(n-1) \rightarrow n$, we have

$$
\begin{aligned}
\frac{d}{d x} \frac{1}{h_{n}(x)} & =\frac{d}{d x} \frac{\bar{F}_{n}(x)}{f_{n}(x)}=\frac{d}{d x} \frac{\bar{F}_{n-1}(x)\left[1-\lambda_{n-1}+\lambda_{n-1} \bar{F}_{n-1}(x)\right]}{f_{n-1}(x)\left[1-\lambda_{n-1}+2 \lambda_{n-1} \bar{F}_{n-1}(x)\right]} \\
& =\left(\frac{d}{d x} \frac{\bar{F}_{n-1}(x)}{f_{n-1}(x)}\right) \frac{1-\lambda_{n-1}+\lambda_{n-1} \bar{F}_{n-1}(x)}{1-\lambda_{n-1}+2 \lambda_{n-1} \bar{F}_{n-1}(x)} \\
& +\frac{\lambda_{n-1} \bar{F}_{n-1}(x)\left[\lambda_{n-1}-1\right]}{\left[1-\lambda_{n-1}+2 \lambda_{n-1} \bar{F}_{n-1}(x)\right]^{2}}
\end{aligned}
$$

Because $F_{n-1} \in \mathcal{C}_{3}$, we have

$$
\begin{gathered}
\lim _{x \rightarrow \infty} \frac{1-\lambda_{n-1}+\lambda_{n-1} \bar{F}_{n-1}(x)}{1-\lambda_{n-1}+2 \lambda_{n-1} \bar{F}_{n-1}(x)}=1 \quad \text { and } \\
\lim _{x \rightarrow \infty} \frac{\lambda_{n-1} \bar{F}_{n-1}(x)\left[\lambda_{n-1}-1\right]}{\left[1-\lambda_{n-1}+2 \lambda_{n-1} \bar{F}_{n-1}(x)\right]^{2}}=0
\end{gathered}
$$

Therefore, we get that $\lim _{x \rightarrow \infty} \frac{d}{d x} \frac{1}{h_{n}(x)}=0$.

By mathematical induction, we conclude the proof. 


\section{Appendix B}

The second partial derivatives of the log-likelihood function are

$$
\begin{aligned}
& \frac{\partial^{2} \mathcal{L}}{\partial \theta_{s} \partial \theta_{j}}=\sum_{i=1}^{n} \frac{1}{\left[f_{n-1}\left(x_{i} ; \rho_{n-2}\right)\right]^{2}}\left\{f_{n-1}\left(x_{i} ; \rho_{n-2}\right) \frac{\partial^{2}}{\partial \theta_{s} \partial \theta_{j}} f_{n-1}\left(x_{i} ; \rho_{n-2}\right)\right. \\
& \left.-\left[\frac{\partial}{\partial \theta_{s}} f_{n-1}\left(x_{i} ; \rho_{n-2}\right)\right]\left[\frac{\partial}{\partial \theta_{j}} f_{n-1}\left(x_{i} ; \rho_{n-2}\right)\right]\right\} \\
& -\frac{1}{\left[1+\lambda_{n-1}-2 \lambda_{n-1} F_{n-1}\left(x_{i} ; \rho_{n-2}\right)\right]^{2}} \\
& \times\left\{2 \lambda_{n-1} \frac{\partial^{2}}{\partial \theta_{s} \partial \theta_{j}} F_{n-1}\left(x_{i} ; \rho_{n-2}\right)\right. \\
& \left.\left.+4 \lambda_{n-1}^{2}\left[\frac{\partial}{\partial \theta_{s}} F_{n-1}\left(x_{i} ; \rho_{n-2}\right)\right]\left[\frac{\partial}{\partial \theta_{j}} F_{n-1}\left(x_{i} ; \rho_{n-2}\right)\right]\right)\right\}, j, s=\overline{1, k} \\
& \frac{\partial^{2} \mathcal{L}}{\partial \theta_{s} \partial \lambda_{j}}=\sum_{i=1}^{n} \frac{1}{\left[f_{n-1}\left(x_{i} ; \rho_{n-2}\right)\right]^{2}}\left\{f_{n-1}\left(x_{i} ; \rho_{n-2}\right) \frac{\partial^{2}}{\partial \theta_{s} \partial \lambda_{j}} f_{n-1}\left(x_{i} ; \rho_{n-2}\right)\right. \\
& \left.-\left[\frac{\partial}{\partial \theta_{s}} f_{n-1}\left(x_{i} ; \rho_{n-2}\right)\right]\left[\frac{\partial}{\partial \lambda_{j}} f_{n-1}\left(x_{i} ; \rho_{n-2}\right)\right]\right\} \\
& -\frac{1}{\left[1+\lambda_{n-1}-2 \lambda_{n-1} F_{n-1}\left(x_{i} ; \rho_{n-2}\right)\right]^{2}}\left\{2 \lambda_{n-1} \frac{\partial^{2}}{\partial \theta_{s} \partial \lambda_{j}} F_{n-1}\left(x_{i} ; \rho_{n-2}\right)\right. \\
& \left.\left.+4 \lambda_{n-1}^{2}\left[\frac{\partial}{\partial \theta_{s}} F_{n-1}\left(x_{i} ; \rho_{n-2}\right)\right]\left[\frac{\partial}{\partial \lambda_{j}} F_{n-1}\left(x_{i} ; \rho_{n-2}\right)\right]\right)\right\}, \\
& j=\overline{0, n-2}, s=\overline{1, k}
\end{aligned}
$$

where

$$
\begin{aligned}
& \frac{\partial^{2}}{\partial \theta_{s} \partial \lambda_{j}} f_{n}\left(x_{i} ; \rho_{n-1}\right)=-2 \lambda_{n-1} \frac{\partial}{\partial \theta_{s}} F_{n-1}\left(x_{i} ; \rho_{n-2}\right) \frac{\partial}{\partial \lambda_{j}} f_{n-1}\left(x_{i} ; \rho_{n-2}\right) \\
& +\left[1+\lambda_{n-1}-2 \lambda_{n-1} F_{n-1}\left(x_{i} ; \rho_{n-2}\right)\right] \frac{\partial^{2}}{\partial \lambda_{j} \partial \theta_{s}} f_{n-1}\left(x_{i} ; \rho_{n-2}\right) \\
& -2 \lambda_{n-1}\left[\frac{\partial}{\partial \theta_{s}} f_{n-1}\left(x_{i} ; \rho_{n-2}\right) \frac{\partial}{\partial \lambda_{j}} F_{n-1}\left(x_{i} ; \rho_{n-2}\right)\right. \\
& \left.+f_{n-1}\left(x_{i} ; \rho_{n-2}\right) \frac{\partial^{2}}{\partial \theta_{s} \partial \lambda_{j}} F_{n-1}\left(x_{i} ; \rho_{n-2}\right)\right]
\end{aligned}
$$


and

$$
\begin{aligned}
& \frac{\partial^{2}}{\partial \theta_{s} \partial \lambda_{j}} F_{n}\left(x_{i} ; \rho_{n-1}\right)=-2 \lambda_{n-1} \frac{\partial}{\partial \theta_{s}} F_{n-1}\left(x_{i} ; \rho_{n-2}\right) \frac{\partial}{\partial \lambda_{j}} F_{n-1}\left(x_{i} ; \rho_{n-2}\right) \\
& +\left[1+\lambda_{n-1}-2 \lambda_{n-1} F_{n-1}\left(x_{i} ; \rho_{n-2}\right)\right] \frac{\partial^{2}}{\partial \theta_{s} \partial \lambda_{j}} F_{n-1}\left(x_{i} ; \rho_{n-2}\right), \quad j \neq n-1 \\
& \frac{\partial^{2} \mathcal{L}}{\partial \theta_{s} \partial \lambda_{n-1}}=\sum_{i=1}^{n} \frac{\frac{\partial}{\partial \theta_{s}} F_{n-1}\left(x_{i} ; \rho_{n-2}\right)}{\left[1+\lambda_{n-1}-2 \lambda_{n-1} F_{n-1}\left(x_{i} ; \rho_{n-2}\right)\right]^{2}} \\
& \times\left\{1-\lambda_{n-1}+2 \lambda_{n-1} F_{n-1}\left(x_{i} ; \rho_{n-2}\right)\right\}, s=\overline{1, k} \\
& \frac{\partial^{2} \mathcal{L}}{\partial \lambda_{r} \partial \lambda_{j}}=\sum_{i=1}^{n} \frac{1}{\left[f_{n-1}\left(x_{i} ; \rho_{n-2}\right)\right]^{2}}\left\{f_{n-1}\left(x_{i} ; \rho_{n-2}\right) \frac{\partial^{2}}{\partial \lambda_{r} \partial \lambda_{j}} f_{n-1}\left(x_{i} ; \rho_{n-2}\right)\right. \\
& \left.-\left[\frac{\partial}{\partial \lambda_{r}} f_{n-1}\left(x_{i} ; \rho_{n-2}\right)\right]\left[\frac{\partial}{\partial \lambda_{j}} f_{n-1}\left(x_{i} ; \rho_{n-2}\right)\right]\right\} \\
& -\frac{1}{\left[1+\lambda_{n-1}-2 \lambda_{n-1} F_{n-1}\left(x_{i} ; \rho_{n-2}\right)\right]^{2}}\left\{2 \lambda_{n-1} \frac{\partial^{2}}{\partial \lambda_{r} \partial \lambda_{j}} F_{n-1}\left(x_{i} ; \rho_{n-2}\right)\right. \\
& \left.\left.+4 \lambda_{n-1}^{2}\left[\frac{\partial}{\partial \lambda_{r}} F_{n-1}\left(x_{i} ; \rho_{n-2}\right)\right]\left[\frac{\partial}{\partial \lambda_{j}} F_{n-1}\left(x_{i} ; \rho_{n-2}\right)\right]\right)\right\}, \quad j, r=\overline{0, n-2}
\end{aligned}
$$

where

$$
\begin{aligned}
& \frac{\partial^{2}}{\partial \lambda_{r} \partial \lambda_{j}} f_{n}\left(x_{i} ; \rho_{n-1}\right)=-2 \lambda_{n-1} \frac{\partial}{\partial \lambda_{r}} F_{n-1}\left(x_{i} ; \rho_{n-2}\right) \frac{\partial}{\partial \lambda_{j}} f_{n-1}\left(x_{i} ; \rho_{n-2}\right) \\
& +\left[1+\lambda_{n-1}-2 \lambda_{n-1} F_{n-1}\left(x_{i} ; \rho_{n-2}\right)\right] \frac{\partial^{2}}{\partial \lambda_{j} \partial \lambda_{r}} f_{n-1}\left(x_{i} ; \rho_{n-2}\right) \\
& -2 \lambda_{n-1}\left[\frac{\partial}{\partial \lambda_{r}} f_{n-1}\left(x_{i} ; \rho_{n-2}\right) \frac{\partial}{\partial \lambda_{j}} F_{n-1}\left(x_{i} ; \rho_{n-2}\right)+f_{n-1}\left(x_{i} ; \rho_{n-2}\right)\right. \\
& \left.\times \frac{\partial^{2}}{\partial \lambda_{r} \partial \lambda_{j}} F_{n-1}\left(x_{i} ; \rho_{n-2}\right)\right], \quad r=\overline{0, n-2}
\end{aligned}
$$

and 


$$
\begin{aligned}
& \frac{\partial^{2}}{\partial \lambda_{r} \partial \lambda_{j}} F_{n}\left(x_{i} ; \rho_{n-1}\right)=-2 \lambda_{n-1} \frac{\partial}{\partial \lambda_{r}} F_{n-1}\left(x_{i} ; \rho_{n-2}\right) \frac{\partial}{\partial \lambda_{j}} F_{n-1}\left(x_{i} ; \rho_{n-2}\right) \\
& +\left[1+\lambda_{n-1}-2 \lambda_{n-1} F_{n-1}\left(x_{i} ; \rho_{n-2}\right)\right] \frac{\partial^{2}}{\partial \lambda_{r} \partial \lambda_{j}} F_{n-1}\left(x_{i} ; \rho_{n-2}\right), \quad k \neq n-1 \\
& \frac{\partial \mathcal{L}}{\partial \lambda_{j} \partial \lambda_{n-1}}=\sum_{i=1}^{m} \frac{-2 \frac{\partial}{\partial \lambda_{j}} F_{n-1}\left(x_{i} ; \rho_{n-2}\right)}{\left[1+\lambda_{n-1}-2 \lambda_{n-1} F_{n-1}\left(x_{i} ; \rho_{n-2}\right)\right]^{2}} \\
& \times\left\{1+2 \lambda_{n-1}-4 \lambda_{n-1} F_{n-1}\left(x_{i} ; \rho_{n-2}\right)\right\}, j \neq n-1 \\
& \frac{\partial^{2} \mathcal{L}}{\partial^{2} \lambda_{n-1}}=\sum_{i=1}^{m} \frac{-\left[1-2 F_{n-1}\left(x_{i} ; \rho_{n-2}\right)\right]^{2}}{\left[1+\lambda_{n-1}-2 \lambda_{n-1} F_{n-1}\left(x_{i} ; \rho_{n-2}\right)\right]^{2}}
\end{aligned}
$$

\section{References}

[1] Aarset M.V., How to identify a bathtub hazard rate, IEEE Transactions on Reliability, 36, (1987).

[2] Akgul F., Frangopol DM, Computational platform for predicting lifetime system reliability profites for different structure types in a network, Journal of Computing in Civil Engineering, 18(2) (2004), 92-104.

[3] Andrews D.F., Herzberg A.M., Data: a collection of problems from many fields for the student and research worker, Springer Science Business Media (2012).

[4] Arnold B.C., Balakrishnan N. and Nagaraja H.N., A First Course in Order Statistics, John Wiley \& Sons, New York (1992).

[5] Asadi M., Bayramoglu I., Mean Residual Life function of a k-out-of-n structure at the system level, IEEE Transactions on Reliability, 55(2) (2006).

[6] Ashour Samir K., Eltehiwy Mahmoud A., Exponentiated power Lindley distribution, Journal of Advanced Research, 6(6) (2015), 895-905.

[7] Băncescu, I., Comparing the expected system lifetimes of k-out-of-m systems using transmuted- $G$ distributions, Proceedings of the Romanian Academy- accepted paper (2017). 
[8] Boland, P. J., Samaniego, F. J., The Signature of a Coherent System and its Applications in Reliability, In: Soyer, R., Mazzuchi, T., Singpurwalla, N. (ed) Mathematical Reliability: An Expository Perspective, Kluwer Academic Publishers, Boston (2004), 129.

[9] Este, AC, Frangopol DM, Repair optimization of highway bridges using system reliability approach, Journal of Structural Engineering, 125(7) (1999), 766-75.

[10] Leadbetter M.R., Lindgren G. and Rootz'en H., Extremes and Related Properties of Random Sequences and Processes, Springer Verlag, New York (1987).

[11] Merovci F., Transmuted Exponentiated Exponential distribution, Mathematical Sciences and Applications E-Notes, 1(2) (2013), 112-122.

[12] Merovci F., Puka L., Transmuted Pareto distribution, ProbStat Forum, 7 (2014), 1-11.

[13] Preda V., Băncescu I., A new family of distributions with a general generic distribution for reliability studies. Log-concavity and Application, International Journal of Risk Theory, Alexandru Myller Publishing Iasi, 1(6) (2016), 13-38.

[14] Samaniego F. J., System signatures and their applications in Engineering Reliability, Springer (2007).

[15] Nadarajah S., Aryal G., On the skew uniform distribution, Random Operators and Stochastic Equations, 12(4) (2004).

[16] Nadar M.O, Frangopol DM, Redundancy of structural systems with and without maintenance: An approach based on lifetime functions, Reliability Engineering and System Safety, 95 (2010), 520-533.

[17] Shaked, M. and Shanthikumar, J.G., Stochastic Orders, Springer Series in Statistics, Springer (2007).

[18] Shaw, W. and Buckley, I., The alchemy of probability distributions: beyond Gram-Charlier expansions and a skew-kurtotic-normal distribution from a rank transmutation map (2007), arXiv prereprint, arXiv, 0901.0434, http://arxiv.org/abs/0901.0434.

Irina Băncescu,

PhD School of Mathematics,

University of Bucharest,

Str. Academiei, nr 14, Bucureşti, Romania.

Email: irina_adrianna@yahoo.com 